\title{
Uso de la escala LRINEC en pacientes con infecciones necrosantes en el miembro superior y su correlación con la morbimortalidad ortopédica
}

\author{
Ricardo Allan, Luis Paladino, Adrián Gross, Emiliano Rivas \\ Equipo de Cirugía de Miembro Superior, Sanatorio de la Trinidad San Isidro, Buenos Aires, Argentina
}

\begin{abstract}
RESUMEN
Objetivo: Utilizar la escala LRINEC en pacientes con infecciones necrosantes de miembros superiores y evaluar su correlación con la morbimortalidad ortopédica. Materiales y Métodos: Se llevó a cabo una revisión sistemática de las historias clínicas de los pacientes operados por nuestro equipo, entre el 1 de marzo de 2015 y el 1 de marzo de 2020 . Se registraron los puntajes de la escala LRINEC de cada paciente operado con diagnóstico clínico y posoperatorio de infección necrosante de partes blandas, así como sus antecedentes clínicos, el microorganismo, las complicaciones y la morbimortalidad ortopédica, y otros datos clínicos importantes (tiempo de internación en terapia intensiva, necesidad de asistencia respiratoria mecánica y de diálisis, cantidad de cirugías), y se los comparó con el puntaje. Resultados: Se analizaron 4126 historias clínicas de pacientes operados por nuestro equipo. Tres tuvieron infecciones necrosantes del miembro superior. El puntaje aplicado en forma retrospectiva determinó que todos tenían una alta probabilidad de sufrir una infección necrosante. Los pacientes con puntaje más alto desarrollaron más comorbilidades ortopédicas y clínicas. Conclusiones: La escala LRINEC es un instrumento reproducible para el diagnóstico de infecciones necrosantes de partes blandas y está relacionada con el número de complicaciones y la morbilidad ortopédica, aunque no necesariamente con la cantidad de cirugías realizadas.
\end{abstract}

Palabras clave: Infección necrosante; escala LRINEC; morbilidad; fascitis necrosante.

Nivel de Evidencia: II

LRINEC score diagnostic value for necrotizing infections of the upper extremity and correlation with morbidity and mortality in Orthopedics patients

\begin{abstract}
Objective: To use the LRINEC scoring system for necrotizing infections of the upper extremity and study its correlation with morbidity and mortality in Orthopedics patients Materials and Methods: We conducted a systematic review of the medical records of patients operated on by our team between March 1, 2015, and March 1, 2020. Data collection included the LRINEC scores of every patient who underwent surgery and had a clinical and postoperative diagnosis of necrotizing soft tissue infection, as well as their clinical history, causative organism, complications, Orthopedics-related morbidity and mortality data, and other significant clinical data (length of intensive care stay, need for mechanical respiratory assistance, need for dialysis, number of surgeries), which were then compared with their respective LRINEC score. Results: The review included 4126 medical records of patients who had undergone surgery by our team. There were three recorded cases of necrotizing infections in the upper extremity. Their LRINEC scores were retrospectively calculated and all of them showed a high risk of developing a necrotizing infection. The patients with the highest scores developed more Orthopedics and other clinical conditions. Conclusions: The LRINEC score is a reproducible method for the diagnosis of necrotizing soft tissue infections and is related to the number of complications and orthopedic conditions, although not necessarily with the number of surgeries.
\end{abstract}

Key words: Necrotizing infection; LRINEC score; morbidity; necrotizing fasciitis.

Level of Evidence: II

Recibido el 23-3-2020. Aceptado luego de la evaluación el 23-5-2020 • Dr. RICARDO ALLAN • ricardoallan85@ hotmail.com ID https://orcid.org/0000-0002-7184-2649 Cómo citar este artículo: Allan R, Paladino L, Gross A, Rivas E. Uso de la escala LRINEC en pacientes con infecciones necrosantes en el miembro superior y su correlación con la morbimortalidad ortopédica. Rev Asoc Argent Ortop Traumatol 2020;85(4):369-376. https://doi.org/10.15417/issn.1852-7434.2020.85.4.1081 


\section{INTRODUCCIÓN}

Las infecciones necrosantes de partes blandas han tenido diversos nombres y clasificaciones con el paso de los años. Esto demuestra que aún no hay consenso entre los especialistas a la hora de referirse a estas infecciones que, aunque tienen una baja frecuencia, representan un reto multidisciplinario para los profesionales.

Su descripción más antigua proviene de Hipócrates. ${ }^{1,2}$ La expresión "fascitis necrosante" es quizás la más utilizada por los especialistas, y se atribuye a Wilson y Schneir. En los últimos tiempos, la expresión genérica "infecciones necrosantes de partes blandas" se ha vuelto común para describir a estas infecciones necrosantes. ${ }^{3}$

Las infecciones necrosantes de tejidos blandos son provocadas por una variedad de microorganismos que pueden afectar a pacientes de cualquier edad, al margen del estado de salud. Esta emergencia ortopédica se manifiesta con signos inespecíficos, como eritema y edema. ${ }^{4}$

Se necesita un alto índice de sospecha para identificar y tratar, de manera oportuna, este cuadro. El diagnóstico temprano, el desbridamiento, los antibióticos de amplio espectro, un enfoque multidisciplinario y los cuidados críticos son vitales para lograr resultados favorables. ${ }^{5-7}$

Varios autores han propuesto herramientas destinadas a diferenciar las infecciones de partes blandas (celulitis y abscesos) de las infecciones necrosantes. Una de las más conocidas es la escala LRINEC (Laboratory Risk Indicator for Necrotizing Fasciitis) desarrollada por Wong y cols., en 2004. ${ }^{8}$ Consiste en la evaluación de seis parámetros de laboratorio y la generación de un puntaje sobre la base de estos parámetros. Un puntaje de 6 o más se interpreta como alta probabilidad de desarrollar una infección necrosante. Hay pocos estudios que evalúan la correspondencia de este puntaje con la morbimortalidad de los pacientes; además, algunos autores han cuestionado el sistema de puntaje de la escala LRINEC para evaluar el pronóstico., ${ }^{910}$

El objetivo de este estudio fue aplicar, en forma retrospectiva, la escala LRINEC en pacientes con infecciones necrosantes del miembro superior y evaluar su correlación con la morbimortalidad ortopédica.

\section{MATERIALES Y MÉTODOS}

Se llevó a cabo una revisión sistemática de las historias clínicas de los pacientes operados por nuestro equipo, en un período de cinco años, desde el 1 de marzo de 2015 hasta el 1 de marzo de 2020. Los criterios de inclusión fueron: pacientes de ambos sexos, de cualquier edad, operados por infecciones necrosantes de partes blandas en los miembros superiores. Los criterios de exclusión fueron: pacientes con necrosis de partes blandas secundaria a cirugías, de causas químicas y por quemaduras.

Se reunió información de los análisis bioquímicos, la historia clínica y las fotografías de los pacientes, y se calculó el puntaje LRINEC (Tabla 1) antes del primer tratamiento quirúrgico. Se registraron los antecedentes clínicos, el agente etiológico, las complicaciones y la morbimortalidad ortopédica de los pacientes, así como también el tiempo de internación en terapia intensiva, la necesidad de asistencia respiratoria mecánica y de diálisis, y la cantidad de cirugías, y se los comparó con el puntaje.

\section{Tabla 1. Escala LRINEC}

\begin{tabular}{|l|c|c|}
\hline Variable & Concentración & Puntos \\
\hline Proteína C reactiva $(\mathrm{mg} / \mathrm{l})$ & $>150$ & 4 \\
\hline Leucocitos (cél./pl) & $<15.000$ & 0 \\
\hline & $15.000-25.000$ & 1 \\
\hline Hemoglobina $(\mathrm{g} / \mathrm{dl})$ & $>25.000$ & 2 \\
\hline & $>13,5$ & 0 \\
\hline & $11-13,5$ & 1 \\
\hline Sodio sérico $(\mathrm{mmol} / \mathrm{l})$ & $<11$ & 2 \\
\hline Creatinina sérica $(\mathrm{mg} / \mathrm{dl})$ & $<135$ & 2 \\
\hline Glucosa sérica $(\mathrm{mg} / \mathrm{dl})$ & $>1,6$ & 2 \\
\hline
\end{tabular}




\section{RESULTADOS}

Se analizaron 4126 historias clínicas de pacientes sometidos a cirugía por nuestro equipo. Se identificaron tres casos tratados por fascitis necrosante (edad 36, 61 y 71 años, respectivamente). Los puntajes de la escala LRINEC aplicados en forma retrospectiva se detallan en la Tabla 2. El agente etiológico fue Streptococcus pyogenes en todos los casos.

Tabla 2. Análisis bioquímicos y escala LRINEC

\begin{tabular}{|c|c|c|c|c|c|c|c|}
\hline & $\begin{array}{c}\text { Proteína C } \\
\text { reactiva (mg/l) }\end{array}$ & $\begin{array}{l}\text { Leucocitos } \\
\text { (cél./ } / \mu \mathrm{l})\end{array}$ & $\begin{array}{l}\text { Hemoglobina } \\
\text { (g/dl) }\end{array}$ & $\begin{array}{l}\text { Sodio sérico } \\
(\mathrm{mmol} / \mathrm{l})\end{array}$ & $\begin{array}{l}\text { Creatinina } \\
\text { sérica (mg/dl) }\end{array}$ & $\begin{array}{c}\text { Glucosa } \\
\text { sérica }(\mathrm{mg} / \mathrm{dl})\end{array}$ & $\begin{array}{l}\text { LRI- } \\
\text { NEC }\end{array}$ \\
\hline Paciente 1 & 237,3 & 6900 & 15 & 129 & 1,05 & 174 & 6 \\
\hline Paciente 2 & 259 & 19600 & 12,6 & 129 & 1,43 & 500 & 9 \\
\hline Paciente 3 & 245 & 23300 & 15,6 & 136 & 2,23 & 259 & 8 \\
\hline
\end{tabular}

La morbimortalidad ortopédica se debió a la desarticulación de un miembro (paciente 3), la amputación de dos dedos y la necesidad de colgajos por defecto de cobertura de partes blandas (paciente 2), la necesidad de injerto de piel (paciente 1), la necesidad de diálisis ( 2 casos) y la necesidad de asistencia respiratoria mecánica (1 caso). El resto de los datos registrados se muestran en la Tabla 3.

Tabla 3. Morbimortalidad y escala LRINEC

\begin{tabular}{|c|c|c|c|c|c|c|c|}
\hline & $\begin{array}{l}\text { LRI- } \\
\text { NEC }\end{array}$ & $\begin{array}{l}\text { Días en } \\
\text { Terapia } \\
\text { Intensiva }\end{array}$ & Muerte & Diálisis & $\begin{array}{l}\text { Asistencia } \\
\text { respiratoria } \\
\text { mecánica }\end{array}$ & $\begin{array}{l}\text { Cantidad de } \\
\text { intervenciones }\end{array}$ & $\begin{array}{l}\text { Morbilidad } \\
\text { ortopédica }\end{array}$ \\
\hline Paciente 1 & 6 & 6 & No & No & No & 3 & Injerto de piel \\
\hline Paciente 2 & 9 & 10 & No & Sí & No & 7 & $\begin{array}{c}\text { Amputación de } \\
\text { falanges más colgajos }\end{array}$ \\
\hline Paciente 3 & 8 & 84 & No & Sí & Sí & 3 & $\begin{array}{c}\text { Desarticulación } \\
\text { del miembro superior }\end{array}$ \\
\hline
\end{tabular}

Las imágenes clínicas se muestran en las Figuras 1-3.

Todos los pacientes tuvieron un puntaje que identificó retrospectivamente la alta probabilidad de sufrir una infección necrosante. Los pacientes con un puntaje más alto desarrollaron más comorbilidades ortopédicas y clínicas. Todos sufrían un dolor desproporcionado en el momento del diagnóstico. 


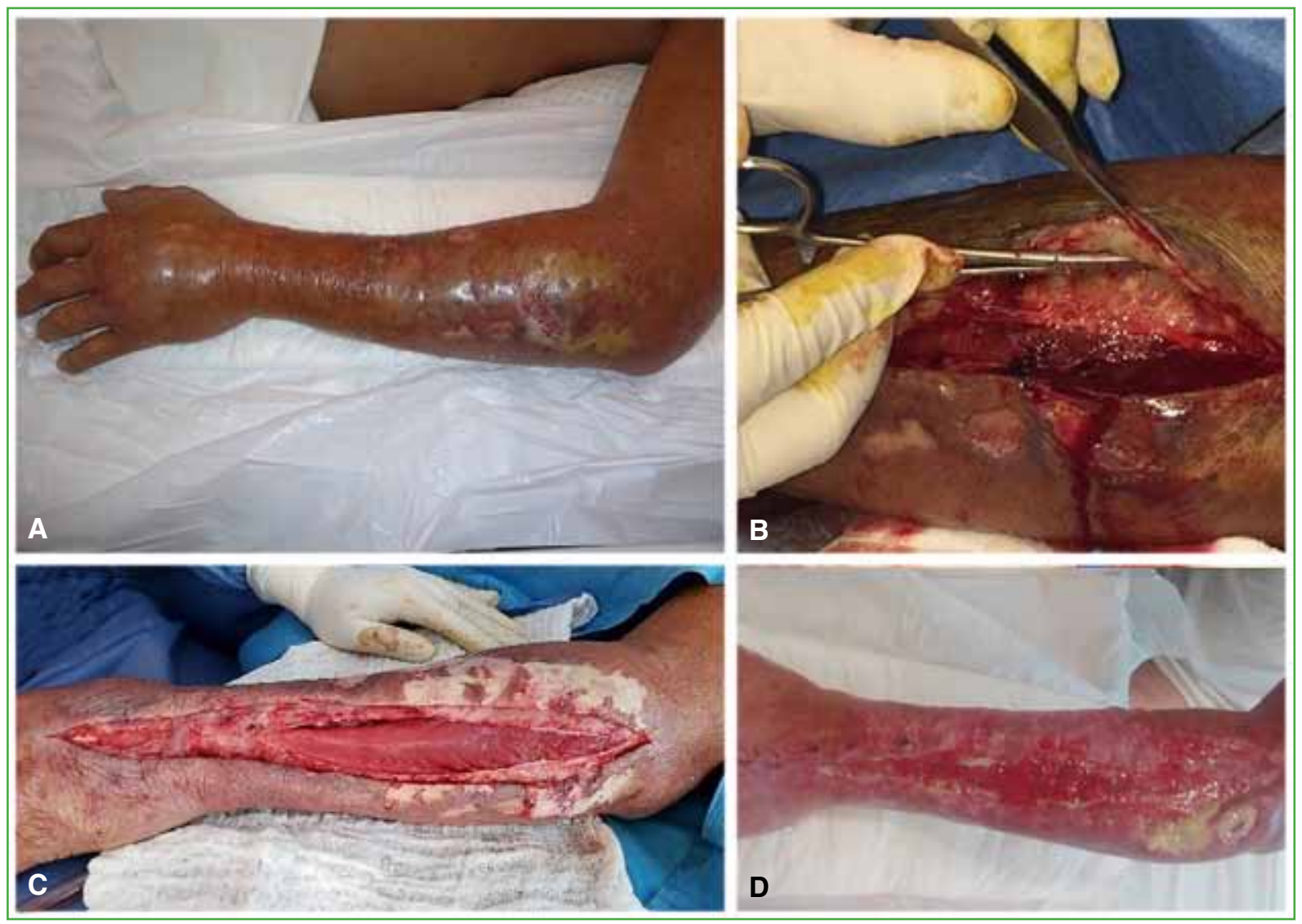

Figura 1. Paciente 1. Hombre de 69 años, con antecedente de cáncer de vejiga, positivo para los virus de la inmunodeficiencia humana y de la hepatitis C; puntaje LRINEC 6. A. Obsérvese el edema de partes blandas que típicamente sobrepasa al eritema en estas infecciones. B. Signo característico de las fascitis necrosantes. Se genera una fina capa de líquido que diseca el tejido celular subcutáneo del plano fascial. C y D. Evolución hasta la granulación para la colocación posterior del injerto de piel.

\section{DISCUSIÓN}

La escala LRINEC, desarrollada tomando como base los datos de 89 pacientes con infecciones necrosantes comparados con 314 pacientes que tenían celulitis severa, absceso, o ambos cuadros, se utiliza ampliamente, pero nunca ha sido validada y los propios autores notaron que muchos otros cuadros podrían causar alteraciones similares en los análisis bioquímicos. ${ }^{11}$

En un análisis de 28 pacientes, Holland ${ }^{10}$ halló que el puntaje 6 como número de corte para determinar la presencia de fascitis necrosante no es suficientemente exacto, y propone disminuirlo a 5 y utilizar biopsias para aumentar la especificidad y la sensibilidad del método.

Wilson y Schneir ${ }^{9}$ publicaron un caso de fascitis necrosante por Clostridium y, luego, flora polimicrobiana, con un puntaje LRINEC de 0.

Asimismo, Tsai y cols. ${ }^{12}$ y Chao y cols. ${ }^{13}$ notaron que el puntaje era bastante pobre para distinguir cuadros de infecciones necrosantes causadas por microorganismos del género Vibrio, ya que estos pacientes, a menudo, tenían recuentos leucocitarios más bajos y otros marcadores inflamatorios.

En un estudio de cohorte, Liao y cols. ${ }^{14}$ evaluaron a 233 pacientes con infecciones necrosantes y 3155 pacientes con celulitis no necrosante, y observaron que el puntaje por sí solo tiene una baja utilidad para diferenciar, en forma temprana, celulitis necrosantes de infecciones no necrosantes. 


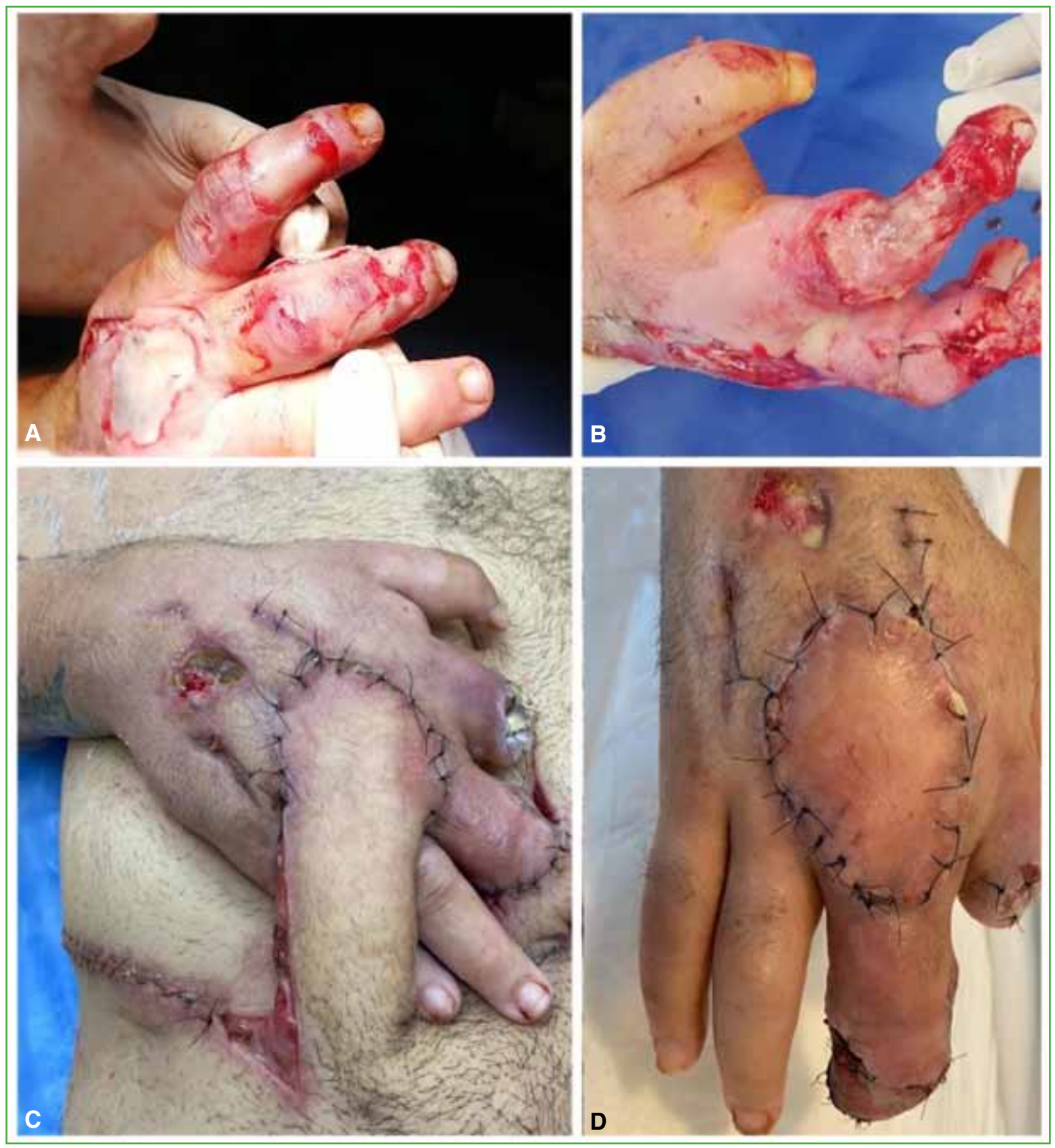

Figura 2. Paciente 2. Hombre de 36 años, con diabetes tipo 1, sin tratamiento; puntaje LRINEC 9. A. Obsérvense la gran lesión de partes blandas y las lesiones necróticas. B. Se desbridó el dorso de la mano y el tejido comprometido en los dedos medio e índice. La evolución fue tórpida y requirió otros desbridamientos, con la pérdida del dedo índice.

C. Por el déficit de cobertura de partes blandas, se realizó un colgajo inguinal para cubrir los defectos. Nótense también los problemas con el muñón del dedo índice. D. Resultado final. 


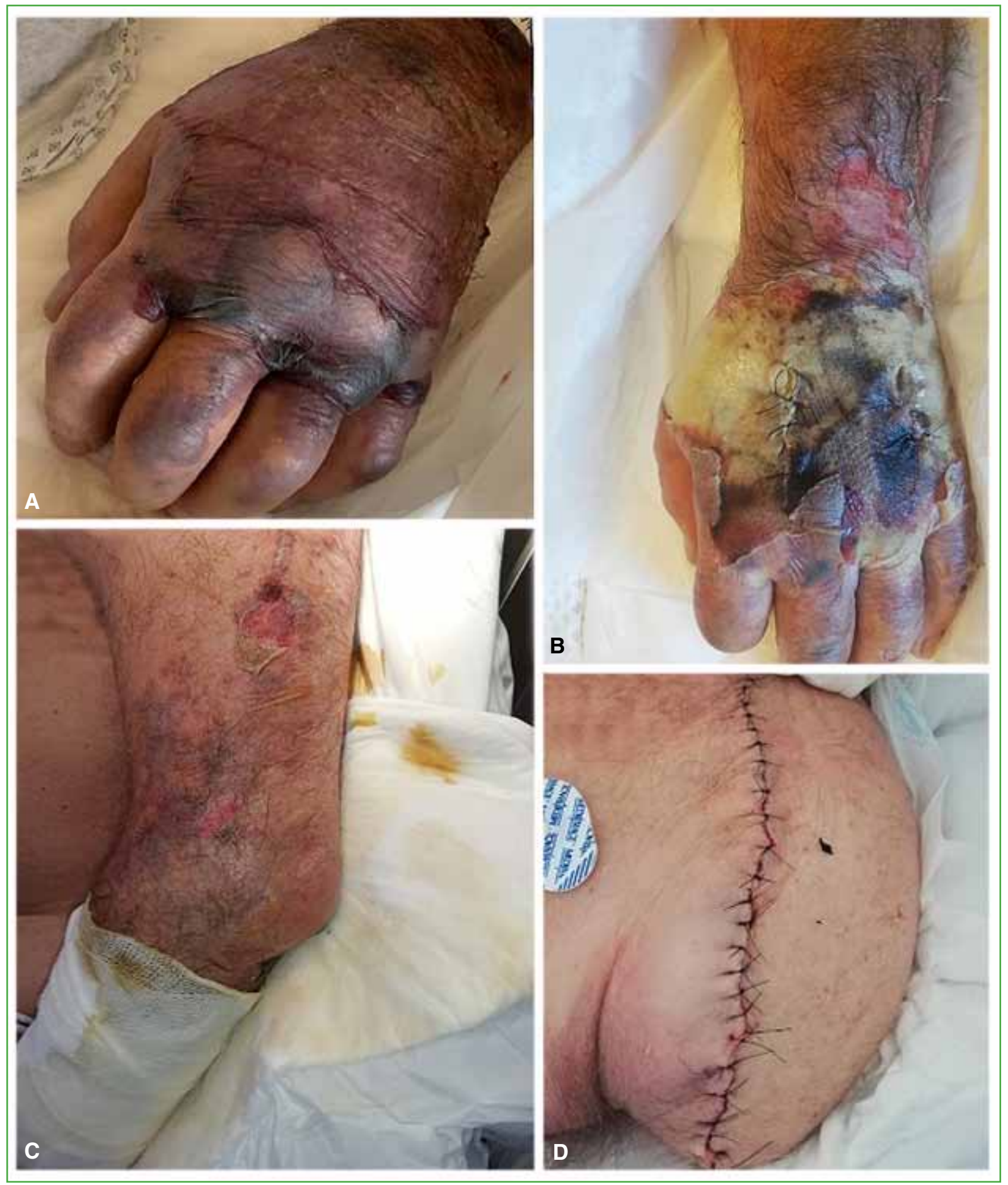

Figura 3. Paciente 3. Hombre de 71 años, con diabetes, insuficiencia renal, marcapasos y enfermedad vascular múltiple; puntaje LRINEC 8. A. Obsérvense las grandes flictenas hemorrágicas, consideradas como un signo de gravedad de la lesión tisular. B. Evolución rápida a la necrosis de partes blandas luego del primer desbridamiento quirúrgico. Se realizó un segundo desbridamiento agresivo. C. Evolución tórpida en pocas horas (6 h), con aumento y avance del edema y las flictenas hemorrágicas en el miembro superior. D. Desarticulación del miembro superior ante la mala evolución clínica. 
Como los autores antes mencionados, Borschitz y cols. ${ }^{15}$ remarcan la necesidad de combinar el puntaje con datos clínicos y no únicamente con los análisis bioquímicos. Los autores proponen una modificación del puntaje agregando puntajes para dolor, fiebre, taquicardia, signos de insuficiencia renal, niveles de fibrinógeno, eritrocitos, y retirar las determinaciones de sodio y glucosa.

En 2017, Bechar y cols. ${ }^{16}$ llevaron a cabo una revisión bibliográfica sistemática de los casos publicados con el puntaje LRINEC desde 2004 hasta 2014, que incluyó 846 pacientes. Los autores determinaron estadísticamente una asociación positiva entre el puntaje y el diagnóstico de fascitis necrosante. También descartan las publicaciones de Tsai y cols., y Chao y cols., por utilizar únicamente el puntaje en gérmenes atípicos, y el estudio de Holland por sesgos metodológicos. Asimismo, señalan la necesidad de realizar más estudios para validar los parámetros adicionales y las modificaciones sugeridos por Borschitz y cols., además de la necesidad de modificar el puntaje LRINEC con datos clínicos (dolor, fiebre, taquicardia) para hacer más específico y sensible el diagnóstico temprano del cuadro en cuestión.

Todos nuestros pacientes fueron diagnosticados correctamente con el puntaje LRINEC. El aumento del puntaje tuvo una incidencia en el número de complicaciones y comorbilidades por encima de 6 puntos, pero no así en la cantidad de cirugías realizadas. El paciente 3 que requirió la desarticulación del miembro superior, asistencia respiratoria mecánica y más días en terapia intensiva no fue el que tuvo el puntaje más alto y el enfermo más crítico de esta serie. Esto nos permite concluir, al igual que otros autores, en que ajustar el puntaje a otros datos clínicos tendría una utilidad pronóstica en este caso y diagnóstica para los casos mencionados en la bibliografía.

Los puntos débiles de nuestro estudio son: la cantidad limitada de pacientes, su naturaleza retrospectiva y que todos sufrieron infecciones necrosantes por Streptococcus pyogenes, no hubo infecciones polibacterianas y anaerobias.

\section{CONCLUSIONES}

La escala LRINEC es un instrumento reproducible para el diagnóstico de infecciones necrosantes de partes blandas y está relacionado con el número de complicaciones y la morbilidad ortopédica, aunque no necesariamente con la cantidad de cirugías realizadas. Es necesario incluir parámetros clínicos al puntaje para poder utilizarlo como factor pronóstico de manera más exacta. La inclusión de valores, como dolor, signos de lesión renal, días de terapia intensiva, podría ser útil y deberá ser evaluada en futuros estudios sobre la asociación del puntaje con la morbilidad ortopédica.

Conflictos de intereses: Los autores no declaran conflictos de intereses.

ORCID de L. Paladino: https://orcid. org/0000-0002-8344-5894

ORCID de A. Gross: https://orcid.org/0000-0002-8287-702X

ORCID de E. Rivas: https://orcid.org/0000-0003-0894-8253

\section{BIBLIOGRAFÍA}

1. Sarani B, Strong M, Pascual J, Schwab CW. Necrotizing fasciitis: current concepts and review of the literature. $J A m$ Coll Surg 2009;208(2):279-88. https://doi.org/10.1016/j.jamcollsurg.2008.10.032

2. Lancerotto L, Tocco I, Salmaso R, Vindigni V, Bassetto F. Necrotizing fasciitis: classification, diagnosis, and management. J Trauma 2012;72(3):560-6. https://doi.org/10.1097/TA.0b013e318232a6b3

3. Tunovic E, Gawazuik J, Bzura T, Embil J, Esmail A, Logsetty S. Necrotizing fasciitis: A six-year experience. J Burn Care Res 2012;33(1):93-100. https://doi.org/10.1097/BCR.0b013e318239d571

4. Lee A, May A, Obremskey WT. Necrotizing soft-tissue infections: an orthopaedic emergency. J Am Acad Orthop Surg 2019;27(5): e199-e206. https://doi.org/10.5435/JAAOS-D-17-00616 
5. Magala J, Makobore P, Makumbi T, Kaggwa S, Kalanzi E, Galukande M. The clinical presentation and early outcomes of necrotizing fasciitis in a Ugandan Tertiary Hospital-a prospective study. BMC Res Notes 2014;7:476. https://doi.org/10.1186/1756-0500-7-476

6. Misiakos EP, Bagias G, Patapis P, Sotiropoulos D, Kanavidis P, Machairas A. Current concepts in the management of necrotizing fasciitis. Front Surg 2014;1:36. https://doi.org/10.3389/fsurg.2014.00036

7. Shiroff AM, Herlitz GN, Gracias VH. Necrotizing soft tissue infections. J Intensive Care Med 2014;29(3):138-44. https://doi.org/10.1177/0885066612463680

8. Wong C-H, Khin L-W, Heng K-S, Tan K-C, Low C-O. The LRINEC (laboratory risk indicator for necrotizing fasciitis) score: a tool for distinguishing necrotising fasciitis from other soft-tissue infections. Crit Care Med 2004; 32(7):1535-41. https://doi.org/10.1097/01.ccm.0000129486.35458.7d

9. Wilson MP, Schneir AB. A case of necrotizing fasciitis with a LRINEC score of zero: clinical suspicion should trump scoring systems. J Emerg Med 2013;44(5):928-31. https://doi.org/10.1016/j.jemermed.2012.09.039

10. Holland MJ. Application of the laboratory risk indicator in necrotising fasciitis (LRINEC) score to patients in a tropical tertiary referral centre. Anaesth Intensive Care 2009; 37(4): 588-92. https://doi.org/10.1177/0310057X0903700416

11. Hakkarainen TW, Kopari NM, Pham TN, Evans HL. Necrotizing soft tissue infections: review and current concepts in treatment, systems of care, and outcomes. Curr Probl Surg 2014; 51: 344-62. https://doi.org/10.1067/j.cpsurg.2014.06.001

12. Tsai Y-H, Hsu RW-W, Huang K-C, Huang T-J. Laboratory indicators for early detection and surgical treatment of vibrio necrotizing fasciitis. Clin Orthop Relat Res 2010;468(8):2230-7. https://doi.org/10.1007/s11999-010-1311-y

13. Chao W-N, Tsai S-J, Tsai C-F, Su C-H, Chan K-S, Lee Y-T, et al. The Laboratory Risk Indicator for Necrotizing Fasciitis score for discernment of necrotizing fasciitis originated from Vibrio vulnificus infections. J Trauma Acute Care Surg 2012;73(6): 1576-82. https://doi.org/10.1097/TA.0b013e318270d761

14. Liao C-I, Lee Y-K, Su Y-C, Chuang C-H, Wong C-H. Validation of the laboratory risk indicator for necrotizing fasciitis (LRINEC) scorefor early diagnosis of necrotizing fasciitis. Tzu Chi Medical J 2012;24(2):73-6. https://doi.org/10.1016/j.tcmj.2012.02.009

15. Borschitz T, Schlicht S, Siegel E, Hanke E, von Stebut E. Improvement of a clinical score for necrotizing fasciitis: 'Pain out of proportion' and high CRP levels aid the diagnosis. PLoS One 2015;10(7):e0132775. https://doi.org/10.1371/journal.pone.0132775

16. Bechar J, Sepehripour S, Hardwicke J, Filobbos G. Laboratory risk indicator for necrotising fasciitis (LRINEC) score for the assessment of early necrotising fasciitis: a systematic review of the literature. Ann R Coll Surg Engl 2017;99(5):341-6. https://doi.org/10.1308/rcsann.2017.0053 